\title{
Prevention of Thromboembolism in Atrial Fibrillation
}

\section{A Meta-analysis of Trials of Anticoagulants and Antiplatelet Drugs}

\author{
Jodi B. Segal, MD, MPH, Robert L. McNamara, MD, MHS, Marlene R. Miller, MD, \\ Nina Kim, MS, Steven N. Goodman, MD, PhD, Neil R. Powe, MD, MPH, MBA, \\ Karen A. Robinson, MSc, Eric B. Bass, MD, MPH, for the Johns Hopkins Evidence-based \\ Practice Center
}

\begin{abstract}
OBJECTIVE: Appropriate use of drugs to prevent thromboembolism in patients with atrial fibrillation (AF) involves comparing the patient's risk of stroke and risk of hemorrhage. This review summarizes the evidence regarding the efficacy of these medications.
\end{abstract}

METHODS: We conducted a meta-analysis of randomized controlled trials of drugs used to prevent thromboembolism in adults with nonpostoperative AF. Articles were identified through the Cochrane Collaboration's CENTRAL database and MEDLINE until May 1998.

MAIN RESULTS: Eleven articles met criteria for inclusion in this review. Warfarin was more efficacious than placebo for primary stroke prevention (aggregate odds ratio [OR] of stroke $=0.30,95 \%$ confidence interval $[\mathrm{CI}] 0.19,0.48$ ), with moderate evidence of more major bleeding (OR 1.90; 95\% CI 0.89, 4.04). Aspirin was inconclusively more efficacious than placebo for stroke prevention (OR $0.56,95 \% \mathrm{CI}$ $0.19,1.65)$, with inconclusive evidence regarding more major bleeds (OR $0.81,95 \%$ CI $0.37,1.77$ ). For primary prevention, assuming a baseline risk of 45 strokes per 1,000 patientyears, warfarin could prevent 30 strokes at the expense of only 6 additional major bleeds. Aspirin could prevent 17 strokes, without increasing major hemorrhage. In direct comparison, there was evidence suggesting fewer strokes among patients on warfarin than among patients on aspirin (aggregate OR $0.64,95 \%$ CI $0.43,0.96$ ), with only sugges-

Received from the Division of General Internal Medicine (JBS, NRP, EBB, NK, KAR), Division of Cardiology (RLM), Division of Pediatric Cardiology (MRM), Oncology Center-Division of Biostatistics (SNG), Johns Hopkins University School of Medicine, Baltimore, Md; Department of Epidemiology (RLM, NRP), Johns Hopkins University School of Hygiene and Public Health, Baltimore, Md; Baltimore Cochrane Center at University of Maryland (NK, KAR), University of Maryland School of Medicine, Baltimore, $M d$.

Address correspondence and reprint requests to Dr. Segal: 1830 E. Monument St., 8th Floor, Baltimore, MD 21205 (e-mail: jsegal@welch.jhu.edu).

Presented as an oral presentation to the Society for General Internal Medicine, San Francisco, Calif, April 1999. tive evidence for more major hemorrhage (OR 1.60, 95\% CI $0.77,3.35)$. However, in younger patients, with a mean age of 65 years, the absolute reduction in stroke rate with warfarin compared with aspirin was low (5.5 per 1,000 person-years) compared with an older group (15 per 1,000 person-years).

CONCLUSION: In general, the evidence strongly supports warfarin for patients with AF at average or greater risk of stroke. Aspirin may prove to be useful in subgroups with a low risk of stroke, although this is not definitively supported by the evidence.

KEY WORDS: atrial fibrillation; warfarin; anticoagulation; stroke; aspirin.

J GEN INTERN MED 2000;15:56-67.

A trial fibrillation $(\mathrm{AF})$ is an increasingly common cardiac dysrhythmia affecting over 2 million people in the United States. ${ }^{1-3}$ Restoration of sinus rhythm may involve consultation with a cardiologist; however, the prevention of cardioembolic events in patients who remain in AF falls within the domain of the primary care physician. ${ }^{4}$ With a recently estimated 3.9 million office visits yearly for patients with $\mathrm{AF},{ }^{5}$ internists need to aware of the current evidence regarding prevention of thromboembolic disease in order to provide the best care for their patients.

The estimate that only one third of patients in $\mathrm{AF}$ are receiving warfarin is remarkably consistent across studies, ${ }^{4,6-8}$ and has raised concerns that warfarin is greatly underutilized in patients with chronic AF. However, for interventions like warfarin that involve significant trade-offs, clinicians need a reliable synthesis of information on risks and benefits in order to make appropriate recommendations to patients. Therefore, we reviewed all of the randomized controlled trials involving anticoagulants and antiplatelet agents in the prevention of thromboembolism in patients with $\mathrm{AF}$. Our aim was to evaluate the strength of the evidence on the efficacy and safety of the drugs used for the prevention of thromboembolic complications in adults with nonpostoperative $\mathrm{AF}$ and to report this information in a format useful to clinicians. 


\section{METHODS}

\section{Study Design}

We performed a literature synthesis and meta-analysis. Articles were included in this review if they included original data addressing the management of nonpostoperative AF or arterial flutter in adult subjects.

\section{Literature Identification and Search Strategies}

The primary source of citations was the CENTRAL database of the Cochrane Collaboration, a comprehensive collection of controlled trials from 1948 onward. As a secondary source, MEDLINE up to May 1998 was searched to ensure completeness. The "related articles" feature of PubMed also was reviewed, as were recent search results submitted to the Baltimore Cochrane Center, tables of contents of relevant journals, and the programs of recent cardiology meetings. The search strategy used the MeSH terms atrial fibrillation and atrial flutter as subject headings and text words, as well as random allocation, double-blind method, and singleblind method. The publication types were randomized controlled trials and controlled clinical trials.

Abstracts of randomized controlled trials were reviewed independently by two members of the study team. Only articles in English were reviewed; however, the English abstracts of non-English publications were reviewed to assess qualitative consistency with our results.

\section{Data Abstraction}

We developed a form with which to abstract information. The section assessing study quality was created after review of forms used in other meta-analytic studies, 9,10 a literature review, ${ }^{11,12}$ and consultation with members of the Cochrane Collaboration. The final form contained 22 questions assessing quality in five areas: (1) representativeness, or how well the study population was described; (2) bias and confounding; (3) description of therapy, including assessment of whether the groups were treated similarly; (4) outcomes and follow-up; and (5) statistical reporting and interpretation. The score in each category was the percentage of points received out of the total available, with the overall quality score calculated as the average of the categorical scores.

The study characteristics and outcomes sections included questions about subject inclusion and exclusion criteria, subject characteristics, therapeutic protocols, and the numbers of events including stroke, peripheral embolism, major and minor hemorrhage, and death.

Study quality was reviewed independently by two reviewers and differences were resolved by consensus. Quantitative data were abstracted by one primary reviewer and checked for accuracy by a secondary reviewer. The reviewers were not masked as to the author, institution, or journal, as this is unlikely to make a significant difference in the results. ${ }^{13}$

Many studies did not give complete information about compliance, so our results reflect an intention-to-treat analysis. When we say "on warfarin" or "on aspirin," we are referring to the group to which the patient was assigned.

\section{Mathematical Pooling}

The odds ratio (OR) was used as the effect measure. As the number of events was small, the OR is nearly equivalent to the rate ratio and the relative risk of an event. We made a qualitative assessment of the combinability of the studies based on the inclusion and exclusion criteria and the protocols. We weighted the studies on the basis of size and the precision of the estimate within each study. We quantitatively assessed heterogeneity, and we used a fixed-effects model to summarize the evidence when there was no significant quantitative heterogeneity between the studies and a random-effects model when there was. Estimates of the relative rates of the outcomes were pooled using standard methods for combining ORs. We used RevMan 3.1 (from the Cochrane Collaboration) and STATA 5.0 (Stata Corp., College Station, Tex) for calculations. The strength of the evidence was graded according to the system shown in Table 1.

To estimate how many strokes could be prevented at the expense of how many bleeds, we averaged the rates of stroke and rates of hemorrhage in the placebo arm of the primary prevention studies, weighted by the number of person-years in each study. From pooling the ORs, we had an aggregate OR for stroke and aggregate OR for hemorrhage on warfarin compared with placebo, and used these to calculated average stroke and average hemorrhage rates on warfarin. Similar calculations estimated the benefits and risks of aspirin therapy.

A more detailed description of the methods is available in a report prepared for the Agency for Health Care Policy and Research. ${ }^{14}$

Table 1. Grading the Evidence

\section{Grade}

Strong evidence of efficacy

Moderate evidence of efficacy

Suggestive evidence of efficacy Inconclusive evidence of efficacy Strong evidence of lack of efficacy
Odds Ratio (OR) and Confidence Interval (Cl)

OR $<1.0$ and the $99 \%$ CI does not include $1.0(p<.01)$

OR $<1.0$ and the $95 \%$ CI does not include 1.0 , but the $99 \%$ CI does include 1.0

$(.01<p<.05)$

$95 \% \mathrm{CI}$ includes 1.0 in the upper tail and the OR is in a clinically meaningful range 95\% CI is widely distributed around 1.0

$95 \% \mathrm{CI}$ is narrow and around 1.0 
Table 2. Studies of Antithromobotic Therapy for Patients with Atrial Fibrillation*

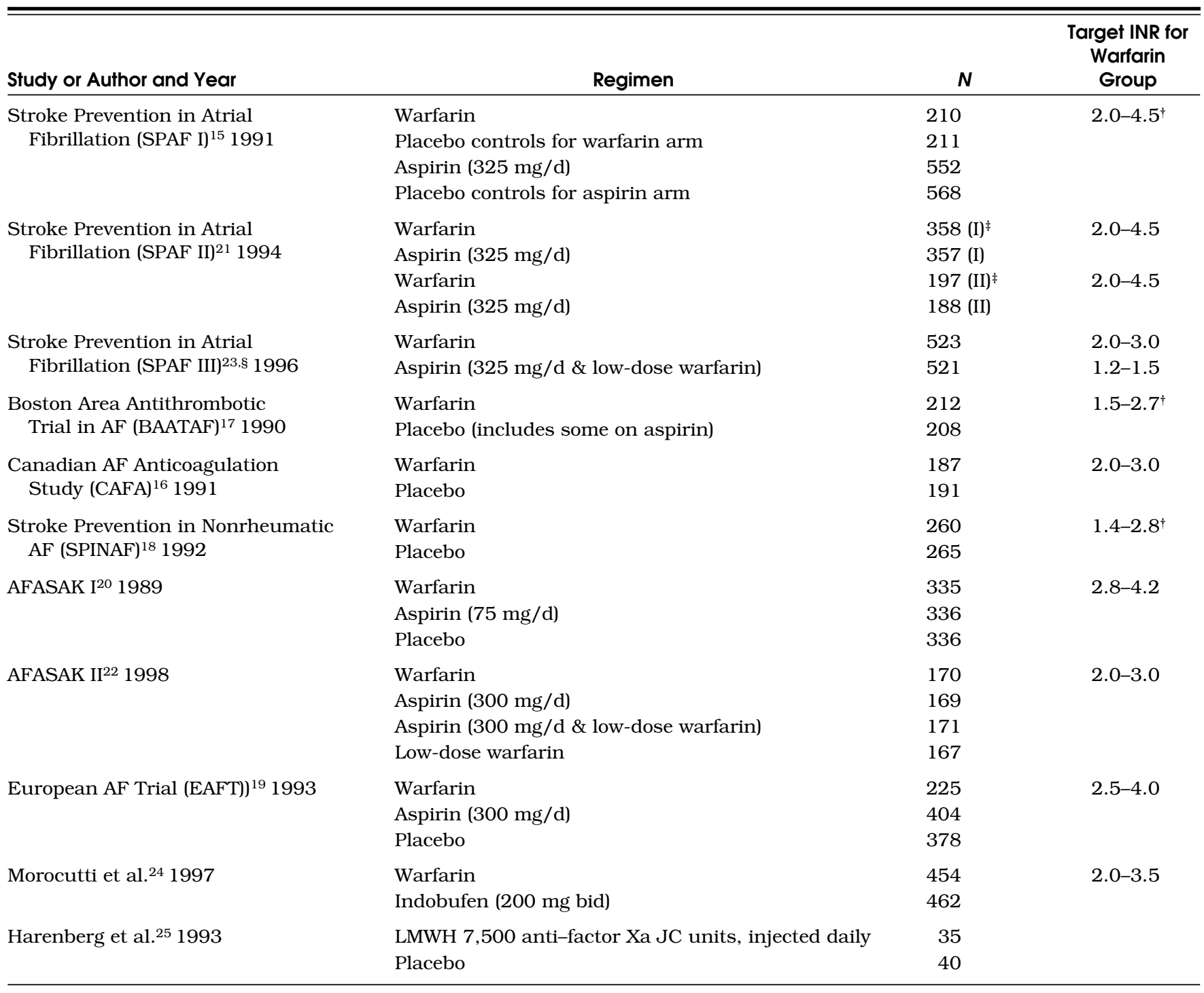

(Continued)

\section{RESULTS}

\section{Literature Yield}

The literature search yielded 36 abstracts for review, including one of a non-English-language article. From these, 11 articles were appropriate for inclusion in this meta-analysis, evaluating warfarin versus placebo, ${ }^{13-18}$ aspirin versus placebo, ${ }^{15,19,20}$ warfarin versus aspirin, ${ }^{20-22}$ warfarin versus aspirin plus low-dose warfarin, ${ }^{22,23}$ warfarin versus indobufen, a reversible cyclo-oxygenase inhibitor, ${ }^{24}$ and low molecular weight heparin versus placebo. ${ }^{25}$

\section{Qualitative Synthesis}

Study Design. Table 2 presents details of the study designs. Importantly, two of the studies, Stroke Prevention in Atrial Fibrillation (SPAF I) ${ }^{15}$ and European Atrial Fibrillation Trial (EAFT), ${ }^{19}$ separated participants into warfarin-eligible and warfarin-ineligible groups on the basis of clinical features or patient preference. Randomization took place after this separation; therefore, the warfarin and aspirin arms of the trials cannot be directly compared.

Quality of the Studies. The studies uniformly provided adequate description of the study participants and excluded subjects, and adequately described the randomization process. They were weakest in their descriptions of ancillary therapies received by the subjects. Notably, a number of the studies were terminated prematurely owing to the high incidence of stroke in the less-intensive arm or, when proof of efficacy was met. This includes the Copenhagen Atrial Fibrillation, Aspirin, and Anticoagulation (AFASAK) $\mathrm{I}^{20}$ and AFASAK $\mathrm{II}^{22}$ studies, SPAF $\mathrm{I}^{15}$ and SPAF III, ${ }^{23}$ the Canadian Atrial Fibrillation Anticoagulation (CAFA) study, ${ }^{16}$ and the Boston Area Anticoagulation Trial for Atrial Fibrillation (BAATAF). ${ }^{17}$ Nearly all of the 
Table 2. (Continued)

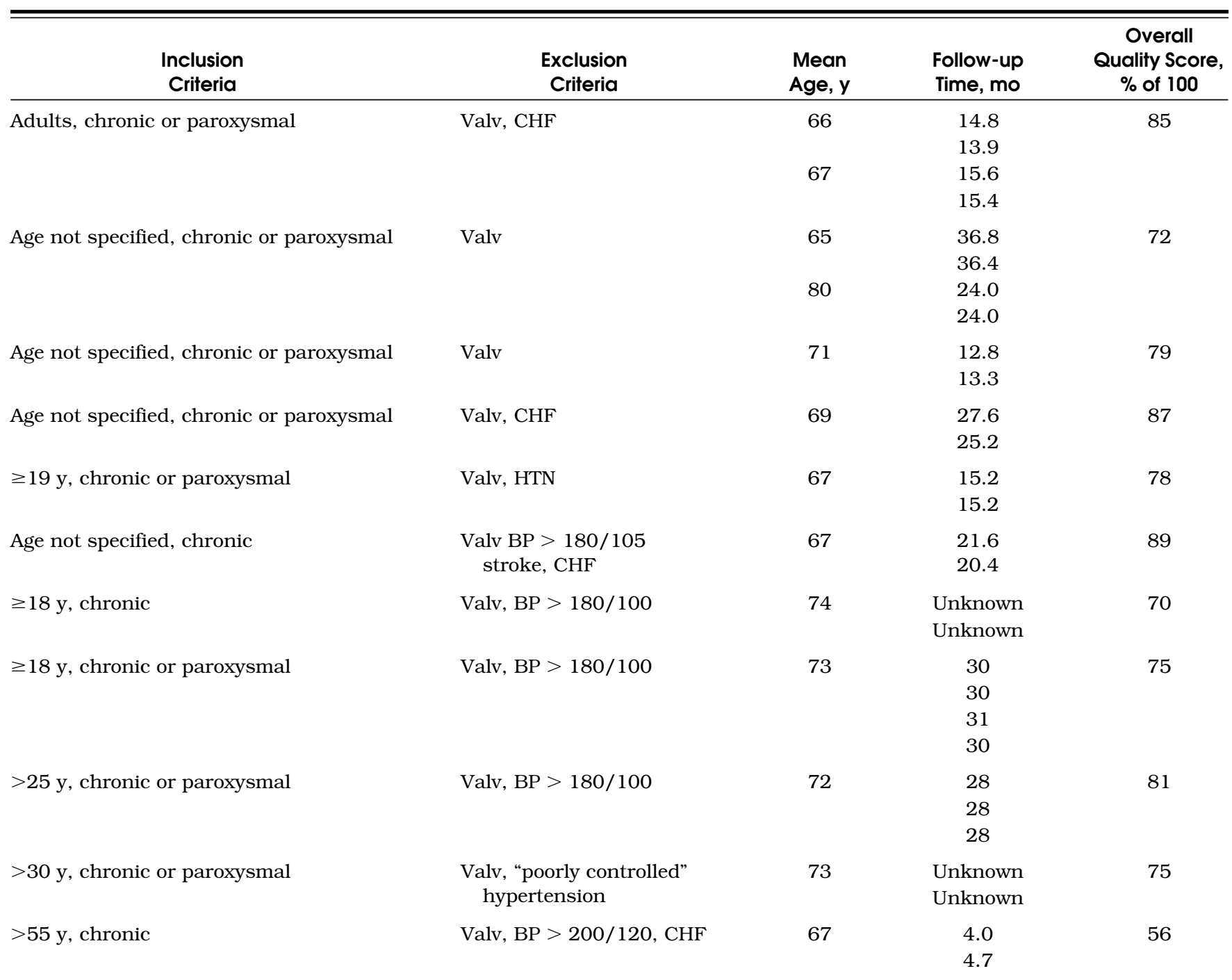

*AF, atrial fibrillation; INR, international normalized ratio; chronic, chronic atrial fibrillation; paroxysmal, paroxysmal atrial fibrillation; BP, blood pressure; Valv, valvular disease; stroke, stroke or transient ischemic attack; CHF, congestive heart failure as defined in each study; LMWH, low molecular weight heparin.

${ }^{\dagger}$ INR is estimated-prothrombin time ratio was used in trial.

¥ Study involves two parallel trials: group I patients are $\leq 75$ years old, group II patients are $>75$ years.

$\S$ Study was restricted to high-risk patients defined as one of the following; systolic blood pressure $160 \mathrm{~mm}$ Hg, history of stroke or transient ischemic attack, ejection fraction $<25 \%$, or recent symptomatic CHF, or female over 75 years.

studies received high overall quality scores; therefore, we did not exclude any study based on its quality score.

Inclusion and Exclusion Criteria for Participants. All of the studies excluded patients with rheumatic valvular disease, for whom there is strong evidence that anticoagulation is indicated. ${ }^{26}$ Importantly, the EAFT trial ${ }^{19}$ and the indobufen study $^{24}$ were secondary prevention trials (i.e., they enrolled patients who had already had a stroke or transient ischemic attack). Thus, the results of these studies were interpreted separately from the other trials. The SPAF III investigators specifically recruited high-risk patients. ${ }^{23}$

Characteristics of the Study Participants. The percentages of participants in each trial with prior stroke, parox- ysmal AF, diabetes, congestive heart failure, and hypertension are listed in Table 3. No trial enrolled exclusively patients with "lone AF" (AF with a structurally normal heart and no significant comorbid illnesses). The trials did not explicitly describe patients' risks of bleeding, but some can be inferred from the patients' clinical characteristics.

Outcomes Reporting. The reporting of outcomes within these studies was satisfactory. The differentiation between hemorrhagic and thromboembolic stroke was nearly always made with imaging studies. The definition of major hemorrhage differed between studies, but was most commonly either a cerebral hemorrhage or a bleed requiring transfusion or hospitalization. 
Table 3. Results of Studies of Antithrombotic Therapies for Patients with Atrial Fibrillation*

\begin{tabular}{|c|c|c|c|c|c|c|c|}
\hline \multicolumn{8}{|c|}{ Patient Characteristics } \\
\hline Study and Year ${ }^{\ddagger}$ & $\begin{array}{l}\text { Mean } \\
\text { Age, } y\end{array}$ & $\begin{array}{l}\text { Male, } \\
N(\%)\end{array}$ & ST, $N(\%)$ & $\begin{array}{l}\text { Parox, } \\
N(\%)\end{array}$ & $\begin{array}{c}\text { DM, } \\
N(\%)\end{array}$ & $\begin{array}{l}\text { CHF, } \\
N(\%)\end{array}$ & $\begin{array}{l}\text { HTN, } \\
N(\%)\end{array}$ \\
\hline $\begin{array}{c}\text { SPAF I }{ }^{15} \\
1991\end{array}$ & 66 & $303(72)$ & $34(8.1)$ & $151(36)$ & $66(16)$ & $70(17)$ & $219(52)$ \\
\hline $\begin{array}{c}\text { SPAF I }{ }^{15} \\
1991\end{array}$ & 67 & $790(71)$ & $73(6.5)$ & $376(34)$ & $196(18)$ & $224(20)$ & $588(52)$ \\
\hline $\begin{array}{l}\mathrm{SPAF} \mathrm{II}^{21} \\
1994\end{array}$ & 65 & $539(75)$ & $43(6.0)$ & $233(33)$ & $122(17)$ & $125(17)$ & $378(53)$ \\
\hline $\begin{array}{l}\text { SPAF II }^{21} \\
1994\end{array}$ & 80 & $225(58)$ & 37 (9.6) & $90(23)$ & $50(13)$ & $98(25)$ & $200(52)$ \\
\hline $\begin{array}{l}\text { SPAF III }^{23} \\
1996\end{array}$ & 71 & $629(60)$ & $381(37)$ & $167(16)$ & $191(18)$ & $234(32)$ & $329(32)$ \\
\hline $\begin{array}{c}\text { CAFA }^{16} \\
1991\end{array}$ & 67 & $282(75)$ & $14(3.7)$ & $26(6.9)$ & $45(12)$ & $83(22)$ & $146(39)$ \\
\hline $\begin{array}{l}\text { SPINAF }^{18} \\
1992\end{array}$ & 67 & $525(100)$ & 0 & 0 & $97(18)$ & $160(30)$ & $305(58)$ \\
\hline $\begin{array}{l}\text { AFASAK I } 26 \\
1989\end{array}$ & 74 & $540(54)$ & $58(5.8)$ & 0 & $84(8.3)$ & $521(52)$ & $323(32)$ \\
\hline $\begin{array}{l}\text { AFASAK II }{ }^{22} \\
1998\end{array}$ & 73 & $102(60)$ & $14(9)$ & 0 & 23 (13) & $120(71)$ & $72(42)$ \\
\hline $\begin{array}{l}\text { Harenberg et al. }{ }^{25} \\
\quad 1993\end{array}$ & 67 & $18(24)$ & $21(28)$ & 0 & $24(32)$ & UNK & UNK \\
\hline
\end{tabular}

Complete description of the quality scores of each study is available. ${ }^{14}$ From our qualitative assessment, we felt that the studies were sufficiently similar in design and in patient populations to allow for pooling of the primary prevention studies within each comparison. The results from the secondary prevention studies are described separately in this report, although for the major outcomes we also report the outcomes with their inclusion.

\section{OUTCOMES}

\section{Warfarin Versus Placebo}

Stroke. As shown in Figure 1a, the rates of stroke on warfarin were significantly below the line of equivalency, except in the CAFA study. ${ }^{16}$
The ORs for the studies comparing warfarin and placebo for the prevention of stroke, shown in Figure 2a, ranged from 0.14 (95\% confidence interval [CI] 0.03, 0.64) in the BAATAF study ${ }^{17}$ to $0.67(95 \%$ CI $0.23,1.92)$ in the CAFA study. ${ }^{16}$ In other words, patients in the BAATAF study treated with warfarin had an odds of stroke that was $14 \%$ of the odds of patients treated with placebo. ${ }^{17}$

In the EAFT secondary prevention study, the OR for stroke for warfarin versus placebo was 0.32 (95\% CI 0.18 , 0.56), well within the range of the other studies, despite high rates of stroke in both arms. ${ }^{19}$

The results of the corresponding pooling of the trial effects are presented graphically in Figure 2a. For the warfarin-versus-placebo comparison, five studies (BAATAF, ${ }^{17}$ CAFA, ${ }^{16}$ SPAF I, ${ }^{15}$ AFASAK, ${ }^{20}$ and Stroke Prevention in Nonrheumatic Atrial Fibrillation [SPINAF] ${ }^{18}$ ) were 
Table 3. (Continued)

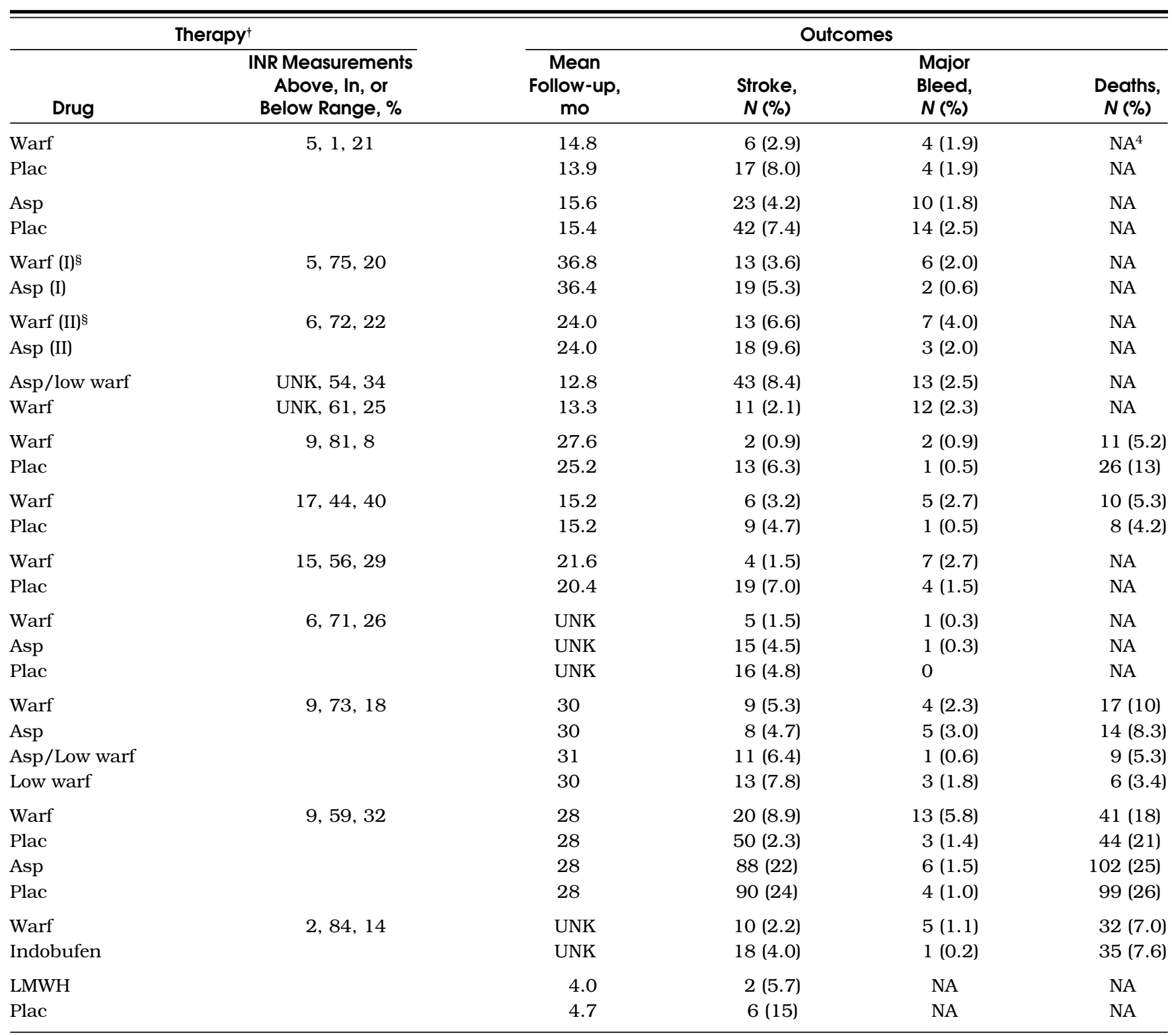

*ST indicates stroke; parox, paroxysmal atrial fibrillation; DM, diabetes mellitus; CHF, congestive heart failure, HTN, hypertension; INR, internatinoal normalized ratio; warf, warfarin; asp, aspirin; plac, placebo; low warf, low-dose warfarin; LMWH, low moleculare weight heparin; NA, not available; UNK, unknown.

${ }^{\dagger}$ Percentage of measurements above, within, or below target INR range.

* Study names are spelled out in Table 2.

$\S_{I}$ is the group with age $\leq 75$ years; II is the group over 75 years.

combined to estimate the effect of warfarin. The total number of subjects on warfarin was 1,204 and on placebo was 1,211 . The aggregate OR of stroke was 0.30 (95\% CI $0.19,0.48$ ), which was strong evidence favoring warfarin over placebo for prevention of stroke. With the inclusion of EAFT, the aggregate OR was 0.34 (95\% CI 0.25, 0.46). ${ }^{19}$

Major Hemorrhage. All studies except the SPAF I ${ }^{15}$ study demonstrated a tendency toward more hemorrhage with warfarin than with placebo (see Fig. 1a).

The ORs for major hemorrhage in the studies comparing warfarin and placebo ranged from a low of 1.0 (95\% CI
$0.25,4.07)$ in SPAF I ${ }^{15}$ to $5.22(95 \%$ CI $0.60,45)$ in the CAFA study $^{16}$. Notably the CAFA participants were above their target international normalized ratio (INR), collectively, for $17 \%$ of the measurements, ${ }^{16}$ while the SPAF I participants were above range for only $5 \%$ of the measurements. ${ }^{15}$

The highest absolute hemorrhage rate occurred in the EAFT secondary-prevention study, with nearly $6 \%$ of the warfarin recipients bleeding during the 28 months of follow-up for a rate of 26 bleeds per 1,000 patient-years. ${ }^{19}$

The rates of cerebral hemorrhage, surprisingly, were not higher in the EAFT study than in the other studies. ${ }^{19}$ No patient on warfarin in this high-risk group had a cerebral 

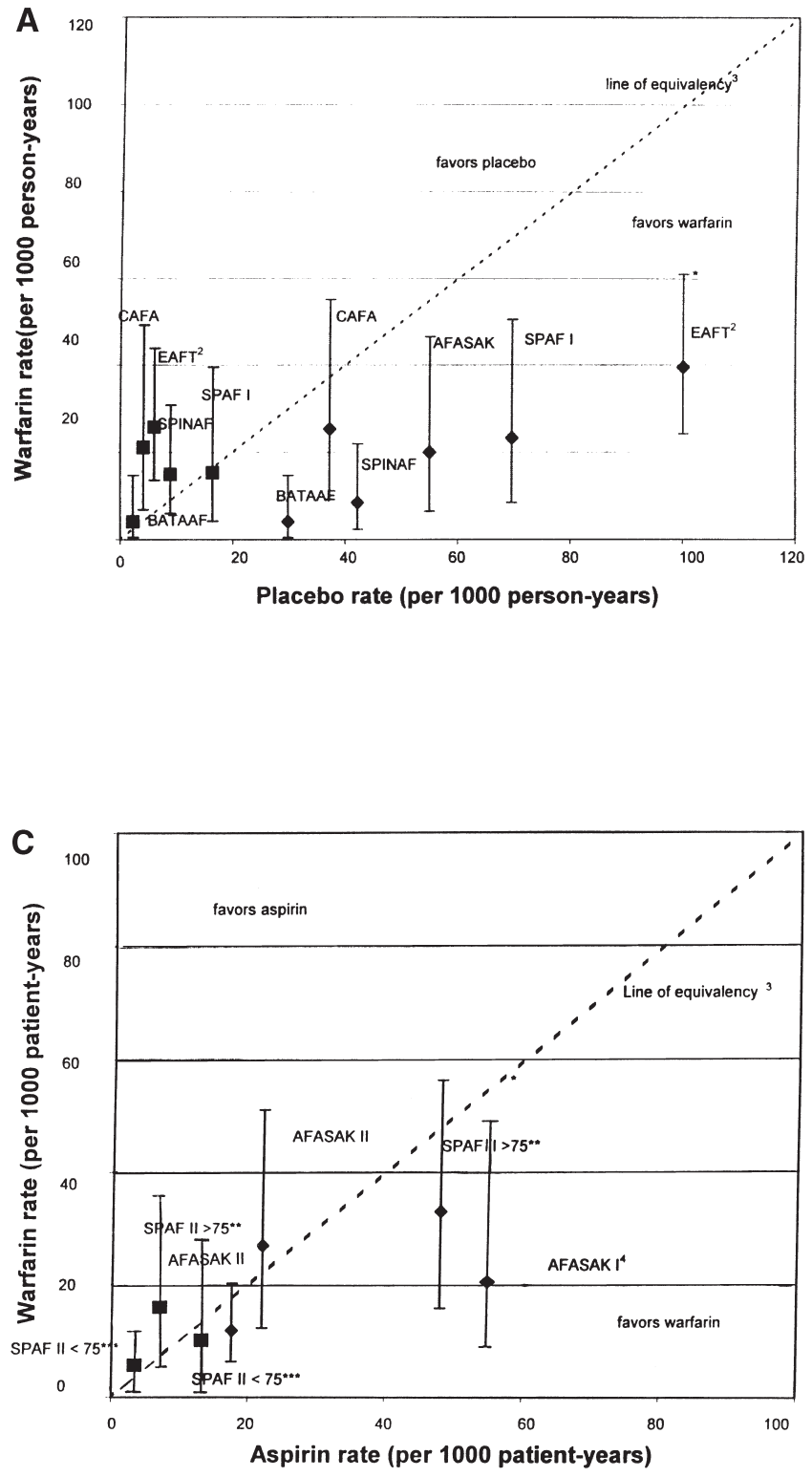

hemorrhage. In the other study with high-risk patients, SPAF III, the rate of cerebral hemorrhage was 5 in 1,000 patient-years. ${ }^{23}$ The groups with the highest incidence of cerebral bleeding was the older subgroup in SPAF II, for whom the rate was 18 in 1,000 patient-years on warfarin. ${ }^{21}$

The aggregate OR for major hemorrhage for the 1,204 subjects on warfarin and the 1,211 subjects on placebo was 1.90 (95\% CI 0.89, 4.04). Thus, there was evidence for more major hemorrhage with warfarin than with placebo. With the inclusion of EAFT, the aggregate OR was 2.35 (95\% CI 1.20, 4.24). ${ }^{19}$

From these studies, the weighted-average stroke rate on placebo was 44 in 1,000 person-years, and the calculated stroke rate on warfarin was 14 in 1,000 person-years. The weighted-average major hemorrhage rate on placebo was 7 in 1,000 person-years, and the calculated rate on warfarin was 13 in 1,000 person-years. Therefore, the literature indicates that warfarin reduced the rate of strokes by

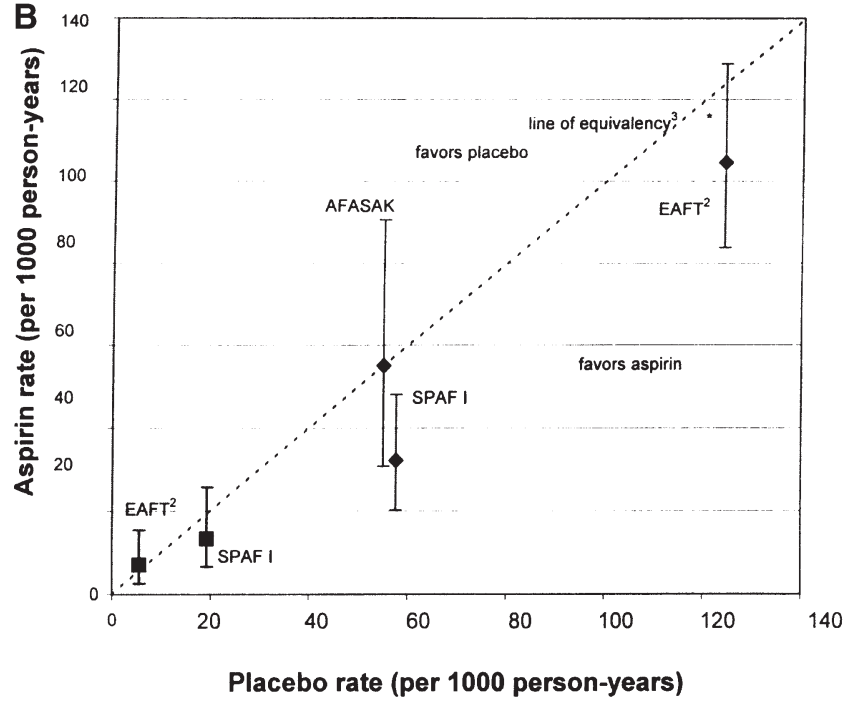

FIGURE la. Rates of stroke and hemorrhage: trials of warfarin versus placebo for patients with atrial fibrillation. 'CAFA indicates Canadian Atrial Fibrillation Anticoagulation study: EAFT, European Atrial Fibrillation Trial; SPAF, Stroke Prevention in Atrial Fibrillation; SPINAF, Stroke Prevention in Nonrheumatic Atrial Fibrillation; BAATAF, Boston Area Anticoagulation Trial in Atrial Fibrillation. 2EAFT was a secondary prevention trial. ${ }^{3}$ Line of equivalency is the line where points would fall if there was no difference in rates between the two treatment arms. *Vertical lines represent $95 \%$ confidence intervals for the rates of stroke and hemorrhage on warfarin. **SPAF $\|>75$ is the group over 75 years old. ***SPAFII $<75$ is the group under 75 years old. Bars with diamonds $(\bullet)$ indicate rates of stroke; bars with squares $(\boldsymbol{\square})$ indicate rates of hemorrhage. (b). Rates of stroke and hemorrhage: trials of aspirin versus placebo for patients with atrial fibrillation. Abbreviations and symbols are explained in the legend to Figure 1a. (c). Rates of stroke and hemorrhage: trials of warfarin versus aspirin for patients with atrial fibrillation. Abbreviations and symbols are explained in the legend to Figure 1a. AFASAK I: data unavailable for yearly hemorrhage rate. ${ }^{4}$

approximately 30 per 1,000 person-years at the expense of 6 major hemorrhages per 1,000 person-years. When EAFT, the secondary prevention trial, was included in these calculations, approximately 40 strokes per 1,000 person-years were prevented with warfarin at the expense of 6 major hemorrhages. ${ }^{19}$

Minor Hemorrhage. There was more minor hemorrhage on warfarin than on placebo in all of the studies. Minor hemorrhage was evaluated in aggregate by combining the four studies that evaluated this outcome (BAATAF, 17 CAFA, ${ }^{16}$ AFASAK I, ${ }^{20}$ and SPINAF ${ }^{18}$ ). The aggregate OR for minor hemorrhage was 2.01 (95\% CI 1.51,2.69), strong evidence for a twofold increased risk of minor hemorrhage on warfarin compared with placebo.

Total Mortality. Mortality was lower on warfarin than on placebo in every trial except CAFA. ${ }^{16}$ The greatest absolute 

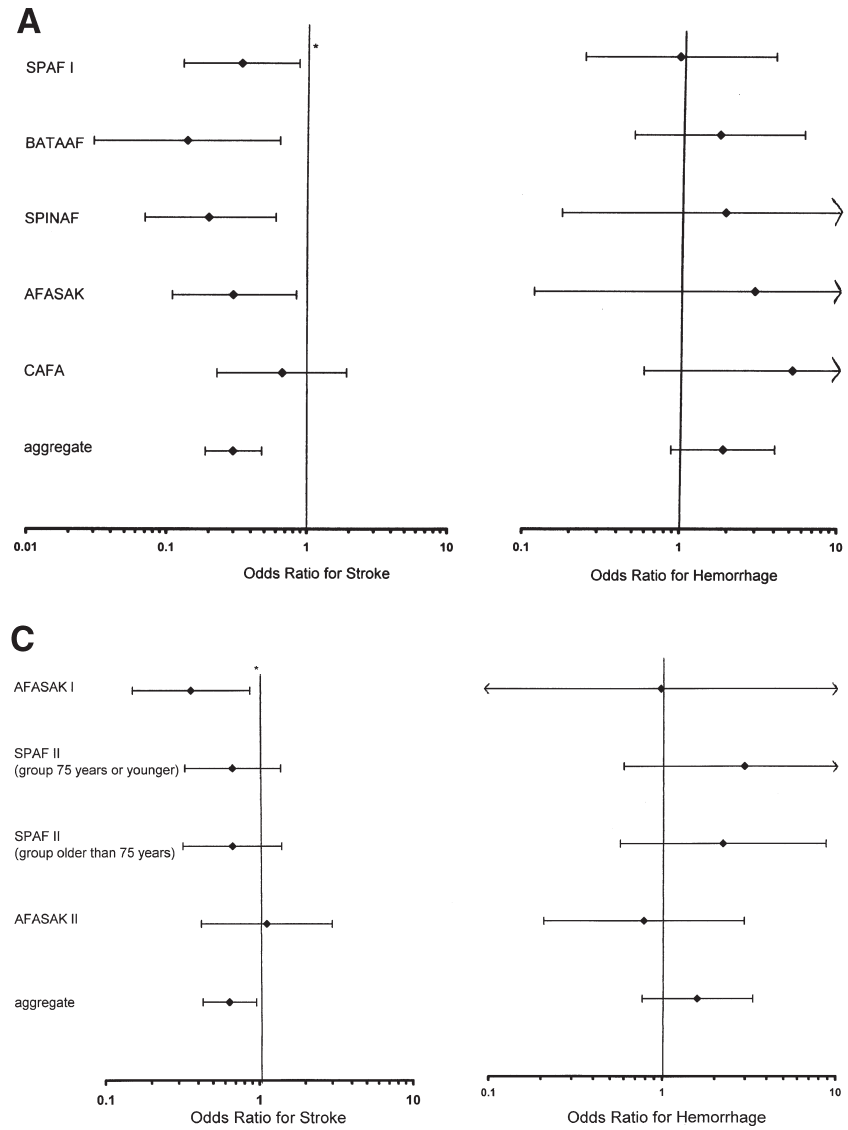

mortality benefit of warfarin compared with placebo was in the BAATAF study, in which the mortality rates were 22 per 1,000 patient-years on warfarin versus 60 per 1,000 patient-years on placebo. ${ }^{17}$ In the secondary prevention trial, EAFT, there was no mortality benefit for warfarin compared with placebo. ${ }^{19}$

For total mortality, three studies were combined (BAATAF, ${ }^{17}$ CAFA, ${ }^{16}$ and SPAF ${ }^{15}$ ), including 609 subjects on warfarin and 610 on placebo. There was a decreased risk of death with warfarin that nearly reached statistical significance, with an aggregate OR of 0.62 (95\% CI $0.38,1.02)$. With the inclusion of EAFT, the aggregate OR was 0.74 (95\% CI $0.53,1.04) .{ }^{19}$

\section{Aspirin Versus Placebo}

Stroke. Three studies evaluated aspirin versus placebo for stroke prevention in patients with AF. Of note, AFASAK $\mathrm{I}^{20}$ used only $75 \mathrm{mg}$ of aspirin daily, whereas SPAF $\mathrm{I}^{15}$ used $325 \mathrm{mg}$ daily. The EAFT secondary prevention study had high stroke rates in both treatment arms as shown on Figure 1b. ${ }^{19}$

The incident stroke rate on aspirin compared with placebo was evaluated by combining the AFASAK $\mathrm{I}^{20}$ and SPAF $I^{15}$ studies using a random effects model, as the warfarin-eligible subgroup of SPAF I was a statistical outlier. ${ }^{27}$ With 888 patients in the aspirin arms and 904 in the placebo arms, the aggregate OR was 0.56 (95\% CI
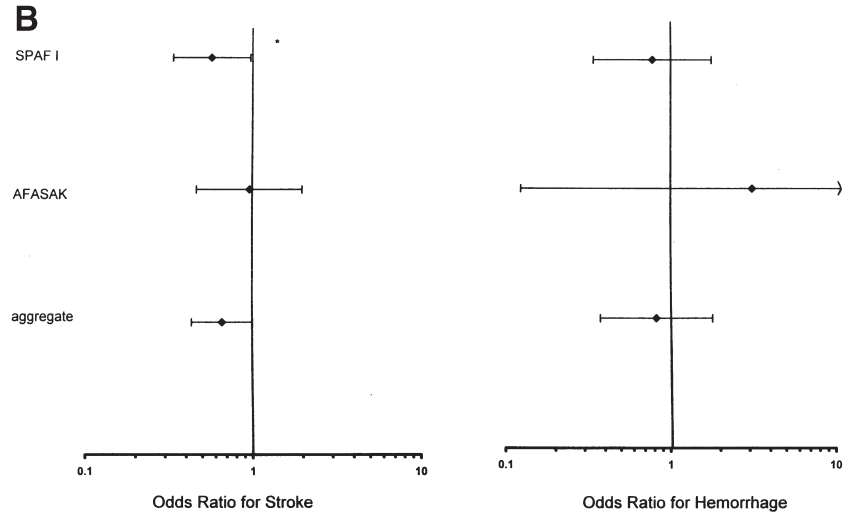

FIGURE 2a. Trials of warfarin versus placebo for patients with atrial fibrillation; odds ratios for stroke and hemorrhage. 'CAFA indicates Canadian Atrial Fibrillation Anticoagulation study: EAFT, European Atrial Fibrillation Trial; SPAF, Stroke Prevention in Atrial Fibrillation; SPINAF, Stroke Prevention in Nonrheumatic Atrial Fibrillation; BAATAF, Boston Area Anticoagulation Trial in Atrial Fibrillation. ${ }^{*}$ Horizontal bars represent $95 \%$ confidence intervals surrounding the point estimates of the odds ratios. (b). Trials of aspirin versus placebo for patients with atrial fibrillation; odds ratios for stroke and hemorrhage. Abbreviations and symbols are explained in the legend to Figure 2a. (c). Trials of warfarin versus aspirin for patients with atrial fibrillation; odds ratios for stroke and hemorrhage. Abbreviations and symbols are explained in the legend to Figure $2 a$.

$0.19,1.65)$, indicating inconclusive evidence of a benefit of aspirin over placebo in primary prevention of stroke in patients with AF. With the inclusion of EAFT, the aggregate OR was 0.76 (95\% CI $0.44,1.33) .{ }^{19}$

Major Hemorrhage. Figure $1 \mathrm{~b}$ shows the rates of major hemorrhages in SPAF $\mathrm{I}^{15}$ and EAFT. ${ }^{19}$ The yearly rate of hemorrhage in the AFASAK $\mathrm{I}^{20}$ study is not depicted, as follow-up time was not explicitly reported for this outcome. In SPAF I, ${ }^{15}$ aspirin did not confer any greater risk of hemorrhage than did placebo (OR 0.73; 95\% CI 0.51,1.22), and there was only suggestive evidence of more bleeding with aspirin in the EAFT trial ${ }^{19}$ (OR $1.41 ; 95 \%$ CI 0.42,4.68). The latter study had surprisingly low hemorrhage rates in both arms, with an aspirin dose of $300 \mathrm{mg}$ daily.

The aggregate OR of major hemorrhage for aspirin compared with placebo, from combining SPAF $\mathrm{I}^{15}$ and AFASAK I, ${ }^{20}$ was 0.81 (95\% CI 0.37, 1.77), which provides inconclusive evidence of bleeding risk from aspirin (Fig. 2b). The AFASAK I study, notably, had only a single event. ${ }^{20}$ Including EAFT, the aggregate OR for major hemorrhage changed little. ${ }^{15}$ Assuming a baseline stroke risk of 50 per 1,000 person-years for untreated patients, the approximate rate in the placebo arms, aspirin could prevent 17 strokes, possibly without major bleeding.

Minor Hemorrhage. Only AFASAK I reported minor hemorrhage, which was rare in both the aspirin and placebo treatment arms. ${ }^{20}$ 
Total Mortality. Only SPAF $\mathrm{I}^{15}$ and $\mathrm{EAFT}^{19}$ reported total mortality. In both studies, there was little difference in mortality rates in the two treatment arms. The OR for death in SPAF I was 0.79 (95\% CI 0.51, 1.22), ${ }^{15}$ and in EAFT the OR was 0.88 (95\% CI 0.66, 1.19). ${ }^{19}$ Thus, there was inconclusive evidence regarding a mortality benefit for aspirin over placebo.

\section{Warfarin Versus Aspirin}

Stroke. Only three studies directly compared warfarin and aspirin, SPAF II, ${ }^{21}$ which stratified subjects into younger and older age groups, AFASAK I, ${ }^{20}$ and AFASAK II ${ }^{22}$ (Fig. 1c).

Among the SPAF II participants, there was the suggestion of a lower rate of stroke on warfarin than on aspirin, for both age groups, despite markedly different rates in the two age groups. ${ }^{21}$ Within SPAF II, the OR for stroke on warfarin compared with aspirin was 0.67 (95\% CI 0.33, 1.38) for the younger age group, with a similar OR and CI for the older age group. ${ }^{21}$ This provides suggestive, although not strong, evidence for a benefit of warfarin over aspirin, over a broad spectrum of risk. The SPAF II group that was under 75 years old had the youngest mean age of any of the trials, 65 years, with very low stroke rates in both arms. ${ }^{21}$

For the pooled analysis of warfarin and aspirin, the results from SPAF $\mathrm{II}^{21}$ were combined with both AFASAK studies, ${ }^{20,22}$ with 1060 participants on warfarin and 1050 on aspirin. For stroke, the aggregate OR ratio was 0.64 (95\% CI 0.43, 0.96). Thus, there was moderate evidence for a decrease in stroke with warfarin compared with aspirin (Fig. 2c).

It is reasonable to believe that aspirin plus low-dose warfarin may be no more efficacious than aspirin alone; therefore, we also present the results when we included the two trials that compared warfarin with aspirin plus a low dose of warfarin (an additional arm of AFASAK II and SPAF III). ${ }^{22,23}$ With inclusion of these two trials, the aggregate OR for stroke for the comparison of warfarin and aspirin was 0.49 (95\% CI 0.36, 0.67).

Major Hemorrhage. The major hemorrhage rate was higher on warfarin than on aspirin in the SPAF II $^{21}$ subjects and among the AFASAK $\mathrm{II}^{22}$ subjects (Fig. 1). The AFASAK I and AFASAK II participants had similar hemorrhage rates in the two treatment arms. In AFASAK II, the hemorrhage rate on warfarin was comparable to that in the other trials, but the hemorrhage rate on aspirin was high. ${ }^{21}$

The aggregate OR for major hemorrhage was 1.60 (95\% CI 0.77, 3.35), indicating only suggestive evidence of an increase in major bleeding on warfarin compared with aspirin. The absolute hemorrhage rates were less than the stroke rates in both trials, in both treatment arms. With inclusion of the two trials that compared warfarin with aspirin plus low-dose warfarin, the aggregate OR for major hemorrhage with warfarin versus aspirin was 1.36 (95\% CI 0.79, 2.33).
Total Mortality. In the SPAF II study, the patients under 75 years old had a greater absolute mortality benefit with warfarin than did the older group. ${ }^{21}$ However, there was a higher percentage of "nonvascular" deaths in the younger group than in the older group, suggesting that these deaths may have been unrelated to therapy. The aggregate OR was 0.96 (95\% CI 0.58, 1.58), strong evidence that there was no overall mortality difference. The result was similar with inclusion of the two trials of low-dose warfarin plus aspirin.

\section{Adjusted-Dose Warfarin Versus Low-Dose Warfarin and Aspirin}

Stroke. The first trial of this comparison, SPAF III, was terminated early because of high stroke rates in the combination arm. ${ }^{23}$ The second trial of warfarin versus the combination of low-dose warfarin and aspirin, AFASAK II, ${ }^{22}$ had a stroke rate in the warfarin-treated patients slightly higher than that in the SPAF III study, while the rate in the combination arm was much lower than that in SPAF III. ${ }^{23}$ This trial was terminated early owing to the results of SPAF III. ${ }^{23}$

The pooling of these data included 693 patients in the adjusted-dose warfarin arm and 692 in the combination therapy arm. The OR for stroke was 0.35 (95\% CI 0.21 , 0.59), strong evidence of a large reduction in the risk of stroke on adjusted-dose warfarin compared with aspirin plus low-dose warfarin, with much of the apparent benefit coming from SPAF III. ${ }^{23}$

Major Hemorrhage. There was a higher rate of hemorrhage on warfarin than on combination therapy in the AFASAK II study, but the aggregate OR for major hemorrhage was 1.14 (95\% CI 0.55, 2.4), inconclusive evidence that there was any difference between the rates of major hemorrhage with adjusted-dose warfarin and with combination therapy. ${ }^{22}$

Minor Hemorrhage and Mortality. The minor hemorrhage rate was higher with warfarin, with an aggregate OR of 1.68 (95\% CI 0.98,2.9). Total mortality was not different between the two groups, with an aggregate OR of 1.02 (95\% CI $0.68,1.5)$.

\section{Warfarin Versus Indobufen and Low Molecular Weight Heparin Versus Placebo}

Stroke. The trial that compared warfarin with indobufen for secondary prevention of stroke in patients with $\mathrm{AF}$ had an OR for stroke of 0.55 (95\% CI 0.24, 1.14)—suggestive evidence favoring warfarin. ${ }^{24}$

Patients on low molecular weight heparin had an OR for stroke of 0.34 (95\% CI 0, 1.62) compared with those on placebo. ${ }^{26}$ The absolute rates were unclear as the follow-up time was not explicitly stated. The relative risk reduction with this drug appeared to be similar to the risk reduction with warfarin, although the evidence only suggested a benefit. 
Major Hemorrhage. In the warfarin-versus-indobufen study, there were more major bleeds on warfarin than on indobufen, although the difference was not statistically significant. ${ }^{24}$ In the low molecular weight heparin study, neither group had any major hemorrhage episodes. ${ }^{25}$

Total Mortality. There was no mortality benefit for warfarin over indobufen. For low molecular weight heparin compared with placebo, evidence suggested a mortality benefit with an OR ratio of death of 0.55 (95\% CI $0.18,1.63)$.

\section{Subgroup Analysis}

The studies ${ }^{16-18}$ with an INR target range with a maximum value below 4.0 had a stroke rate reduction of similar magnitude to that in the studies ${ }^{15,20}$ with higher target INR ranges (OR 0.32, 95\% CI 0.19, 0.56; and OR 0.35, 95\% CI 0.19, 0.65, respectively). The incidence of bleeding with the higher target INRs was not higher than with the lower ranges (OR 2.29, 95\% CI 0.94, 5.53; and OR 1.26, 95\% CI 0.34, 4.70, respectively).

Linear regression of stroke rates versus percentage of measurements below target INR did not suggest that this was an important predictor of events ( $p=0.37$ ).

\section{DISCUSSION}

Essential for evidence-based decision making is an appreciation of the strength of available evidence. Although a number of review articles, ${ }^{25-34}$ meta-analyses, ${ }^{35,36}$ and decision analyse ${ }^{37,38}$ have evaluated the use of warfarin and aspirin, few ${ }^{30,39}$ have objectively graded the strength of the evidence that supports use of these medications.

These trials provided strong evidence that warfarin is more efficacious than placebo in primary prevention of stroke. There was also strong evidence that the same conclusion holds for secondary stroke prevention-the number of strokes prevented with warfarin exceeds the number of major bleeds. Of course, decisions about the use of warfarin need to be individualized for patients at higher risk of bleeding, such as those with alcoholism, renal insufficiency, or a previous gastrointestinal bleed. ${ }^{40}$

The evidence regarding treatment of patients with lone AF is scant, as no trials specifically addressed this population. However, a review of patients with lone $\mathrm{AF}^{32}$ who were enrolled in BAATAF, ${ }^{17}$ SPAF I, ${ }^{15}$ and SPINAF ${ }^{18}$ found low stroke rates for these patients in the placebo arms. Thus, for patients at the lowest risk of stroke, the absolute reduction in risk of stroke with warfarin compared with placebo may be so low that its benefit is offset by the increased risk of bleeding. For such a patient, the use of warfarin depends largely on how the patient views its risks against its benefit.

The evidence allows less-definitive conclusions regarding the efficacy of aspirin in stroke prevention in patients with AF. A recent observational study from the SPAF III investigators aimed to identify a group of patients who may benefit most if treated with aspirin. ${ }^{41}$ They recruited patients without the high-risk features required for inclusion in the SPAF III trial ${ }^{23}$ into an observational study of aspirin therapy in patients with AF. Their observations support the conclusions from the trials-patients at low risk of stroke benefit little from aspirin, as their risk is already low. Although the evidence regarding the bleeding risk on aspirin was inconclusive in these trials, other work suggests this is a risk that needs to be considered. ${ }^{42}$

The next question addressed was whether aspirin is as efficacious as warfarin for primary and secondary prevention of stroke. The evidence from direct comparison is limited; therefore, we draw conclusions from the trials that independently compared warfarin with placebo and aspirin with placebo. Among patients who have average stroke risk, the use of warfarin could prevent 30 strokes at the expense of 6 major bleeds. Aspirin could prevent only 17 strokes, but without increasing major hemorrhage. If we consider the trials that compared warfarin with aspirin plus low-dose warfarin as if they were warfarinversus-aspirin trials, we can more definitively conclude that warfarin is more efficacious than aspirin for stroke prevention. For secondary stroke prevention, warfarin is clearly superior to aspirin.

Other studies of this topic are indicated. Before supporting the routine use of aspirin for primary prevention, attention should be paid to identifying a subgroup of patients for whom aspirin may be appropriate therapy. Also, studies of warfarin versus aspirin and their combined use in lower-risk populations are indicated. A comparison of low molecular weight heparin with warfarin or with aspirin is warranted.

The design features of a clinical trial that result in the best possible outcomes can be replicated to some extent in practice. The optimal INR was studied retrospectively in the EAFT participants who had been randomized to anticoagulation, ${ }^{43}$ as well as in a case-control study of patients with $\mathrm{AF} .{ }^{44}$ Among the EAFT participants, the optimal INR was from 2.0 to $3.9 .{ }^{43}$ In the case-control study, the risk of stroke rose steeply below an INR of 2.0.44 A meta-analysis aimed at defining the relation between adverse events and INR suggests that the optimal INR depends on the patient's risk factors for stroke. ${ }^{45}$ Efforts to refine the management of a patient's INR with the use of anticoagulation clinics may result in better outcomes, ${ }^{46}$ as may home monitoring systems for measuring prothrombin times. ${ }^{47}$

We analyzed the data with the outcomes ascribed to patients in the treatment groups to which they were assigned. Further insights come from reviewing the outcomes according to the actual treatment received. In BAATAF, the two patients that had strokes while assigned to warfarin therapy had subtherapeutic prothrombin time ratios at the time of their events. ${ }^{17}$ In the SPAF I trial, of the six strokes in the warfarin-assigned group, only two occurred in patients with therapeutic prothrombin times. ${ }^{15}$ In AFASAK I, only one of the four strokes occurred in a 
patient sufficiently anticoagulated. ${ }^{20}$ In SPAF III, of the 11 events in patients assigned to warfarin, 3 occurred within days of discontinuing warfarin. ${ }^{23}$ An analysis of anticoagulation intensity during major bleeding complications in the SPAF II trial revealed that 16 of the 34 bleeding patients were within the target range at the time of bleeding, while 13 were above range and 4 were below. ${ }^{48}$ Clearly, compliance affects the outcomes.

Concern about the generalizablility of trial results is always appropriate given the stringent inclusion and exclusion criteria. Furthermore, there is concern that randomization never completely adjusts for the heterogeneity of participants within a trial, and that the outcomes, particularly with binary events, are strongly influenced by the inclusion of a small number of high-risk patients. ${ }^{49}$ For the most part, however, the trials enrolled subjects with $\mathrm{AF}$ and significant comorbidities, as would be expected in practice. Therefore, the results of this pooled analysis should be widely applicable.

Limitations of this meta-analysis are those common to all such studies. Our quality assessment assured us that these were high-quality studies appropriate for inclusion. Our review of the non-English-language literature revealed only one trial, although the possibility of publication bias cannot be discounted. The ability to perform subgroup analyses was limited by the few trials of each drug comparison, and also by the lack of reporting of outcomes by patient characteristics.

We conclude that, in general, the evidence strongly supports use of warfarin in patients with AF who have average or greater risk of stroke. For patients with $\mathrm{AF}$ who have a lower risk of stroke, aspirin may be useful, but the evidence is inconclusive.

The authors thank Dr. Francis Chesley of the Agency for Health Care Policy and Research, and Drs. Hanan Bell and Michael LeFevre from the American Academy of Family Physicians for their helpful suggestions regarding this project; Dr. David Haines from the American College of Cardiology, and Drs. Ronald Berger and Gary Gerstenblith for their expert advice; Dr. David Yu and Mr. Paul Abboud for assistance with data abstraction, and Ms. Donna Lee for extensive help with the manuscript.

This study was conducted by the Johns Hopkins EvidenceB6666ased Practice Center through contract 290-97-006 from the Agency for Health Care Policy and Research, Rockville, Md.

The authors are responsible for the content of this article, including any treatment recommendations. No statement in this article should be construed as an official position of the Agency for Health Care Policy Research or the U.S. Department of Health and Human Services.

\section{REFERENCES}

1. Wolf PA, Mitchell JB, Baker CS, Kannel WB, D'Agostino RB. Impact of atrial fibrillation on mortality, stroke and medical costs. Arch Intern Med. 1998;158:229-34.

2. Feinberg WM, Blackshear JL, Laupacis A, Kronmal R, Hart RG.
Prevalence, age distribution, and gender of patients with atrial fibrillation. Arch Intern Med. 1995;155:469-73.

3. Psaty BM, Manolia TA, Kuller LH, et al. Incidence of and risk factors for atrial fibrillation in older adults. Circulation. 1997;96:2455-61.

4. Stafford RS, Singer DE. National patterns of warfarin use in atrial fibrillation. Arch Intern Med. 1996;156:2537-41.

5. Stafford RS, Robson DC, Misra B, Ruskin J, Singer DE. Rate control and sinus rhythm maintenance in atrial fibrillation: national trends in medication use, 1980-1996. Arch Intern Med. 1998;158:2144-8.

6. Brass LM, Krumholz HM, Scinto JD, Mathur D, Radford M. Warfarin use following ischemic stroke among medicare patients with atrial fibrillation. Arch Intern Med. 1998;158:2093-100.

7. Albers GW, Yim JM, Belew KM, et al. Status of antithrombotic therapy for patients with atrial fibrillation in university hospitals. Arch Intern Med. 1996;156:2311-6.

8. Munschauer FE, Priore RL, Hens M, Castilone A. Thromboembolism prophylaxis in chronic atrial fibrillation: practice patterns in community and tertiary-care hospitals. Stroke. 1997;28:72-6.

9. Bass EB, Powe NR, Goodman SN, et al. Efficacy of immune globulin in preventing complications of bone marrow transplantation; meta-analysis. Bone Marrow Transplant. 1993;12:273-82.

10. Powe NR, Schein OD, Gieser SC, et al. Synthesis of the literature on visual acuity and complications following cataract extraction with intraocular lens implantation. Arch Ophthamol. 1994;112:239-52.

11. Detsky AS, Naylor CD, O'Rourke K, McGeer AJ, L'abbe KA. Incorporating variations in the quality of individual randomized trials into meta-analysis. J Clin Epidemiol. 1992;45(3):255-65.

12. Chalmers TC, Smith H, Blackburn B, et al. A method for assessing the quality of a randomized control trial. Control Clin Trials. 1981;2:31-49.

13. Justice AC, Cho MK, Winker MA, Berlin JA, Rennie D. Does masking author identity improve peer review quality? A randomized controlled trial. PEER Investigators. JAMA. 1998;280(3):240-2.

14. McNamara FL, Miller MR, Segal JB, et al. Evidence Report on Management of New Onset Atrial Fibrillation. Evidence Report/ Technology Assessment No. 1. (Prepared by the Johns Hopkins Evidence-based Practice Center under Contract No. 290-97-006.) Rockville, Md: Agency for Health Care Policy and Research; 1998. AHCPR publication 99-E021.

15. Stroke Prevention in Atrial Fibrillation Investigators. Stroke prevention in atrial fibrillation study. Circulation. 1991;84:527-39.

16. Connolly SJ, Laupacis A, Gent M, Roberts RS, Cairns JA, Joyner C. Canadian Atrial Fibrillation Anticoagulation (CAFA) study. J Am Coll Cardiol. 1991;18:349-55.

17. The Boston Area Anticoagulation Trial for Atrial Fibrillation Investigators. The effect of low-dose warfarin on the risk of stroke in patients with nonrheumatic atrial fibrillation. N Engl J Med. 1990; 323:1505-11.

18. Ezekowitz MD, Bridgers SL, James KE, et al., and the Veterans Affairs Stroke Prevention in Nonrheumatic Atrial Fibrillation Investigators. Warfarin in the prevention of stroke associated with nonrheumatic atrial fibrillation. N Engl J Med. 1992;327:1406-12. Erratum. N Engl J Med. 1993;328:148.

19. EAFT Study Group (European Atrial Fibrillation Trial). Secondary prevention in non-rheumatic atrial fibrillation after transient ischaemic attack or minor stroke. Lancet. 1993;342:1255-62.

20. Petersen P, Boysen G, Godtfredsen J, Andersen ED, Andersen B. Placebo-controlled, randomized trial of warfarin and aspirin for prevention of thromboembolic complications in chronic atrial fibrillation: the Copenhagen AFASAK study. Lancet. 1989;1:175-9.

21. Warfarin versus aspirin for prevention of thromboembolism in atrial fibrillation: Stroke Prevention in Atrial Fibrillation II Study. Lancet. 1994;343:687-91.

22. Gullov AL, Koefoed BG, Peresen P, et al. Fixed minidose warfarin and aspirin alone and in combination vs adjusted-dose warfarin for stroke prevention in atrial fibrillation: Second Copenhagen Atrial Fibrillation, Aspirin, and Anticoagulation Study. Arch Intern Med. 1998;158:1513-21.

23. Adjusted-dose warfarin versus low-intensity, fixed-dose warfarin 
plus aspirin for high-risk patients with atrial fibrillation: Stroke Prevention in Atrial Fibrillation III randomized clinical trial. Lancet. 1996;348:633-8.

24. Morocutti-C, Amabile-G, Fattapposta-F, et al. and the SIFA (Studio Italiano Fibrillazione Atriale) Investigators. Indobufen versus warfarin in the secondary prevention of major vascular events in nonrheumatic AF. Stroke. 1997;28:1015-21.

25. Harenberg J, Weuster B, Pfitzer M, et al. Prophylaxis of embolic events in patients with $\mathrm{AF}$ using low molecular weight heparin. Semin Thromb Hemost. 1993:116-21.

26. Peverill RE, Harper RW, Gelman J, et al. Determinants of increased regional left atrial coagulation activity in patients with mitral stenosis. Circulation. 1996;94:331-9.

27. Stroke Prevention in Atrial Fibrillation Investigators. A differential effect of aspirin on prevention of stroke in atrial fibrillation. $J$ Stroke Cerebrovasc Dis. 1993;3(3):181-8.

28. Wolf PA, Singer DE. Preventing stroke in atrial fibrillation. Am Fam Physician. 1997;56(9):2242-50.

29. Blackshear JL, Kopecky SL, Litin SC, Safford RE, Hammill SC. Management of atrial fibrillation in adults: prevention of thromboembolism and symptomatic treatment. Mayo Clin Proc. 1995;71:150-60.

30. Albers GW. Choice of antithrombotic therapy for stroke prevention in atrial fibrillation. Arch Intern Med. 1998;158:1487-91. Editorial.

31. Ackermann RJ. Anticoagulant therapy in patients aged 80 years or more with atrial fibrillation. Arch Fam Med. 1997;6:105-10. Editorial.

32. Laupacis A, Albers G, Dunn M, Feinberg W. Antithrombotic therapy in atrial fibrillation. Chest. 1992;102(4):426S-32S.

33. Prystowsky EN, Benson DW, Fuster V, et al. Management of patients with atrial fibrillation. Circulation. 1996;93:1262-77.

34. Antiplatelet Trialists' Collaboration. Collaborative overview of randomized trials of antiplatelet therapy. BMJ. 1994;308:81-106.

35. Atrial Fibrillation Investigators. Risk factors for stroke and efficacy of antithrombotic therapy in atrial fibrillation: analysis of pooled data from five randomized controlled trials. Arch Intern Med. 1994;154:1449-57.

36. Atrial Fibrillation Investigators. The efficacy of aspirin in patients with atrial fibrillation. Analysis of pooled data from 3 randomized trials. Arch Intern Med. 1997;157:1237-40.

37. Middlekauff HR, Stevenson WG, Gornbein JA. Antiarrhythmic prophylaxis vs warfarin anticoagulation to prevent thromboembolic events among patients with atrial fibrillation. Arch Intern Med. 1995; 155:913-20.

38. Gage BF, Cardinalli AB, Albers GW, Owens DK. Cost-effectiveness of warfarin and aspirin for prophylaxis of stroke in patients with nonvalvular atrial fibrillation. JAMA. 1995; 274:1839-44.

39. Laupacis A, Albers G, Dalen J, Dunn MI, Jacobson AK, Singer DE. Antithrombotic therapy in atrial fibrillation. Chest. 1998; 114: 579S-89S.

40. McMahan DA, Smith DM, Carey MA, Zhou XH. Risk of major hemorrhage for outpatients treated with warfarin. J Gen Intern Med. 1998; 13:311-6.

41. SPAF Investigators Committee on Echocardiography. Transesophageal echocardiographic correlates of thromboembolism in highrisk patients with nonvalvular atrial fibrillation. Ann Intern Med. 1998;128:639-47.

42. He J, Whelton PK, Vu B, Klag MJ. Aspirin and risk of hemorrhagic stroke. JAMA. 1998;280:1930-5.

43. European Atrial Fibrillation Trial Study Group. Optimal oral anticoagulant therapy in patients with nonrheumatic atrial fibrillation and recent cerebral ischemia. N Engl J Med. 1995;333:5-10.

44. Hylek EM, Skates SJ, Sheehan MA, Singer DE. An analysis of the lowest effective intensity of prophylactic anticoagulation for patients with nonrheumatic atrial fibrillation. N Engl J Med. 1996; 335:5406.

45. Gage BF, Cardinalli AB, Wyrwich KW, Albers GW, Rich MW, Owens DK. Meta-analysis of the optimal INR in nonvalvular atrial fibrillation. J Gen Intern Med. 1998;13(suppl 1):25. Abstract.

46. Chiquette E, Amato MG, Bussey HI. Comparison of an anticoagulation clinic with usual medical care: anticoagulation control, patient outcomes, and health care costs. Arch Intern Med. 1998; 158(15):1641-7.

47. Sawicki PT. A structured teaching and self-management program for patients receiving oral anticoagulation: a randomized controlled trial. JAMA. 1999;281(2):145-50.

48. Stroke Prevention in Atrial Fibrillation Investigators. Bleeding during antithrombotic therapy in patients with atrial fibrillation. Arch Intern Med. 1996;156(4):409-16.

49. Ioannidis JP, Lau J. The impact of high-risk patients on the results of clinical trials. J Clin Epidemiol. 1997;50(10):1089-98. 